\title{
Unusual Fundus Autofluorescence Appearance in a Patient with Hydroxychloroquine Retinal Toxicity
}

\author{
Sleiman Abou-Ltaif \\ East Surrey Hospital, Redhill, UK
}

\section{Key Words}

Hydroxychloroquine - Toxicity · Autofluorescence

\begin{abstract}
Purpose: To report an unusual fundus autofluorescence aspect in a patient with suspected hydroxychloroquine retinal toxicity. Method: Case report of an unusual presentation of a patient treated for 9 consecutive years with a therapeutically recommended dose of hydroxychloroquine. Result: We report the case of a 53-year-old Caucasian female treated with $400 \mathrm{mg}$ hydroxychloroquine for rheumatoid arthritis over 9 years, currently on methotrexate and folinic acid, who stopped treatment 3 years ago. The cumulative dose is estimated at $1.314 \mathrm{~kg}$. She recently noticed a reduction of vision in both eyes to $0.34 \log M A R$, with colour vision problems and major distortion in central vision. Fundus autofluorescence revealed minimal foveal pigmentary changes and more pronounced changes in the retina elsewhere. Foveal changes were confirmed by optical coherence tomography in both eyes. The patient did not report any colour perception difficulties or night vision problems and has no family history of any eye condition. Her visual field tested by an optician was full, with some central changes. Conclusion: Retinal toxicity from hydroxychloroquine can present in a different aspect than the commonly known retinal toxicity, and it happens even after years of cessation of the drug. The role of cumulative dose in toxicity is supported in this paper.
\end{abstract}

(c) 2015 S. Karger AG, Basel

\section{Introduction}

Hydroxychloroquine (Plaquenil) is a chloroquine derivative used in the treatment of many diseases including malaria, rheumatoid arthritis, and systemic lupus erythematosus.

KARGER 125/s $\quad \begin{aligned} & \text { Dr. Sleiman Abou-Ltaif } \\ & \text { East Surrey Hospital } \\ & \text { Canada Avenue } \\ & \text { Redhill RH } 5 \text { RH (UK) } \\ & \text { E-Mail Dr.Sleiman @ gmail.com }\end{aligned}$


Abou-Ltaif: Unusual Fundus Autofluorescence Appearance in a Patient with Hydroxychloroquine Retinal Toxicity

Retinal toxicity from hydroxychloroquine is rare, but even if the medication is discontinued, vision loss may be irreversible and may continue to progress. Before treatment is initiated, a complete ophthalmic examination should be performed to determine any baseline maculopathy. In early toxicity, there are no visible signs, but field, optical coherence tomography (OCT), and multifocal electroretinography (mfERG) changes can be detected [1].

\section{Method}

We report the case of a 53-year-old Caucasian female with a history of rheumatoid arthritis treated with hydroxychloroquine $400 \mathrm{mg}$ daily for 9 years, between 2002 and 2011, who presented with distortion and decrease in vision, colour vision disturbances, and major central vision distortion for the last 2 months before presentation in June 2014.

\section{Results}

Examination of this patient showed a best corrected visual acuity of 0.3 logMAR in both eyes, the Ishihara test revealed red/green colour vision unspecific disturbances, and her fundus examination revealed subtle pigment disturbances in both foveae but otherwise normal looking retina. OCT (fig. 1) revealed subfoveal changes in the outer retina and the retinal pigment epithelium layer. No visual field or electrophysiology testing was performed; however, a visual field test performed by her optician was normal except for few central changes. Fundus autofluorescence (FAF) revealed foveal pigment disturbances and an area of hypoautofluorescence outside the arcade that is symmetrical between the 2 eyes (fig. 2, fig. 3).

\section{Discussion}

Hydroxychloroquine ocular toxicity includes keratopathy, ciliary body involvement, lens opacities, and retinopathy. The incidence of true hydroxychloroquine retinopathy is very low; although its pathogenesis is unclear, risk factors include daily dosage of hydroxychloroquine, cumulative dosage, duration of treatment, coexisting renal or liver disease, patient age, and concomitant retinal disease [2]. Patients usually complain of difficulty with reading, decreased vision, missing central vision, glare, blurred vision, light flashes, and metamorphopsia. They can also be asymptomatic.

Melles and Marmor [3] found that the overall prevalence of hydroxychloroquine retinopathy is $7.5 \%$ but varied with daily consumption [odds ratio, 5.67; $95 \%$ confidence interval (CI), 4.14-7.79 for $>5.0 \mathrm{mg} / \mathrm{kg}$ ] and with duration of use (odds ratio, 3.22; 95\% CI, 2.204.70 for $>10$ years). For a daily consumption of $4.0-5.0 \mathrm{mg} / \mathrm{kg}$, the prevalence of retinal toxicity remained less than $2 \%$ within the first 10 years of use but rose to almost $20 \%$ after 20 years of use. Other major risk factors include kidney disease (odds ratio, 2.08; 95\% CI, 1.443.01) and concurrent tamoxifen citrate therapy (odds ratio, 4.59; 95\% CI, 2.05-10.27) [3].

Greenstein et al. [4] evaluated patients with standard automated perimetry using the 10-2 and/or 24-2 visual field program (Zeiss, Meditec), mfERG, spectral-domain OCT (SDOCT), and short-wavelength FAF. Their findings showed that 44\% (15 patients) had abnormal results on at least 1 test; however, only 3\% (2 patients) had abnormal results on all 4 tests. Excluding short-wavelength FAF, 7 of the 15 patients had abnormal visual fields, 
Abou-Ltaif: Unusual Fundus Autofluorescence Appearance in a Patient with Hydroxychloroquine Retinal Toxicity

mfERG ring ratios, and SD-OCTs. The remaining 8 had abnormal mfERGs and/or visual fields and normal SD-OCTs. The authors found no evidence of abnormal SD-OCTs in the presence of normal mfERG and visual field results. They suggest that functional deficits precede structural changes seen on SD-OCT in these patients [4].

In early stages, mild changes are limited to a pericentral ring of increased FAF. More advanced stages present as pericentral mottled loss of FAF with increased FAF in the adjacent retina and later on a complete loss of pericentral FAF [5].

Lee et al. [6] found that a pericentral pattern of hydroxychloroquine retinopathy was predominant among Korean patients, rather than the traditional (bull's eye) parafoveal pattern of damage, in 8 out of 9 patients diagnosed with toxicity, among 218 patients screened.

The use of mfERG may detect retinal dysfunction in a considerable number of eyes with normal ophthalmoscopy and fluorescein angiography. The higher variability of colour vision and visual field testing results suggests the use of mfERG as a primary screening tool for retinal dysfunction in long-term hydroxychloroquine treatment [7].

In our case, the retinal changes only seen on FAF were very subtle in the foveal and macular area and were mainly in the area outside the arcades, mainly inferiorly, and very symmetrical, and occurred 3 years after cessation of the drug taken at a normal dose, however, for 9 years.

Due to normal fundus appearance apart from foveal changes, the lack of electrodiagnostic testing in our unit, and the patient not being keen on travelling to large centres for testing, we have not requested an ERG or simply an mfERG, which is the main weakness in our case report.

Although we cannot rule out the diagnosis of sectorial retinitis pigmentosa, the normal fundus appearance, normal visual field, absence of night vision problems, and lack of family history are all important factors against such a diagnosis. ERG testing would not show any changes as the area involved is not large enough to reduce the ERG waves. To the best of our knowledge, this aspect has never been reported before, especially in the Caucasian population, and the findings were not confined to the macular area.

\section{Statement of Ethics}

Dr. Sleiman Abou-Ltaif, sole author of this work, certifies that this report complies with the guidelines for human studies and animal welfare regulations. The subject has given her informed consent regarding publishing anonymised data related to her case. The manuscript has not been submitted for approval by the institute's committee on human research as the case is applicable.

\section{Disclosure Statement}

The author has no conflicts of interest with the drug or machines used in this case report.

\section{References}

Hansen MS, Schuman SG: Hydroxychloroquine-induced retinal toxicity. EyeNet Magazine, June 2011.

-2 Yam JC, Kwok AK: Ocular toxicity of hydroxychloroquine. Hong Kong Med J 2006;12:294-304. 
Abou-Ltaif: Unusual Fundus Autofluorescence Appearance in a Patient with Hydroxychloroquine Retinal Toxicity

3 Melles RB, Marmor MF: The risk of toxic retinopathy in patients on long-term hydroxychloroquine therapy. JAMA Ophthalmol 2014;132:1453-1460.

4 Greenstein VC, Amaro-Quireza L, Abraham ES, Ramachandran R, Tsang SH, Hood DC: A comparison of structural and functional changes in patients screened for hydroxychloroquine retinopathy. Doc Ophthalmol 2015;130:13-23.

5 Kellner U, Renner AB, Tillack H: Fundus autofluorescence and mfERG for early detection of retinal alterations in patients using chloroquine/hydroxychloroquine. Invest Ophthalmol Vis Sci 2006;47:35313538.

6 Lee DH, et al: Pericentral hydroxychloroquine retinopathy in Korean patients. Ophthalmology 2015;122:1252-1256.

7 Missner S, Kellner U: Comparison of different screening methods for chloroquine/hydroxychloroquine retinopathy: multifocal electroretinography, color vision, perimetry, ophthalmoscopy, and fluorescein angiography. Graefes Arch Clin Exp Ophthalmol 2012;250:319-325.

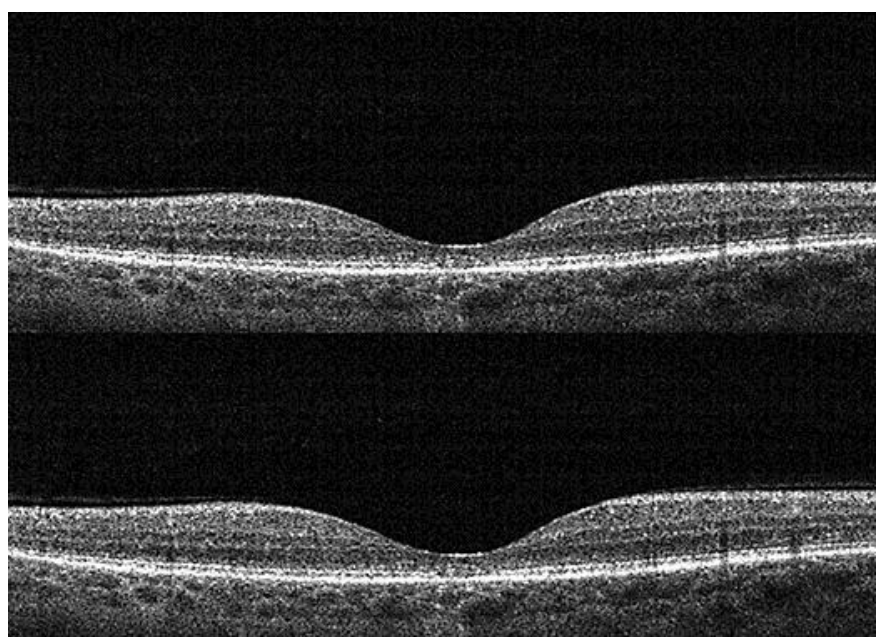

Fig. 1. OCT of the right (top) and left eye (bottom) showing outer retina and retinal pigment epithelium changes and foveal thinning. 


\section{Case Reports in \\ Ophthalmology}

\begin{tabular}{l|l}
\hline \multicolumn{2}{l}{ Case Rep Ophthalmol 2015;6:186-190 } \\
\hline DOI: 10.1159/000433417 & $\begin{array}{l}\text { @ 2015 S. Karger AG, Basel } \\
\text { www.karger.com/cop }\end{array}$ \\
\hline
\end{tabular}

Abou-Ltaif: Unusual Fundus Autofluorescence Appearance in a Patient with Hydroxychloroquine Retinal Toxicity

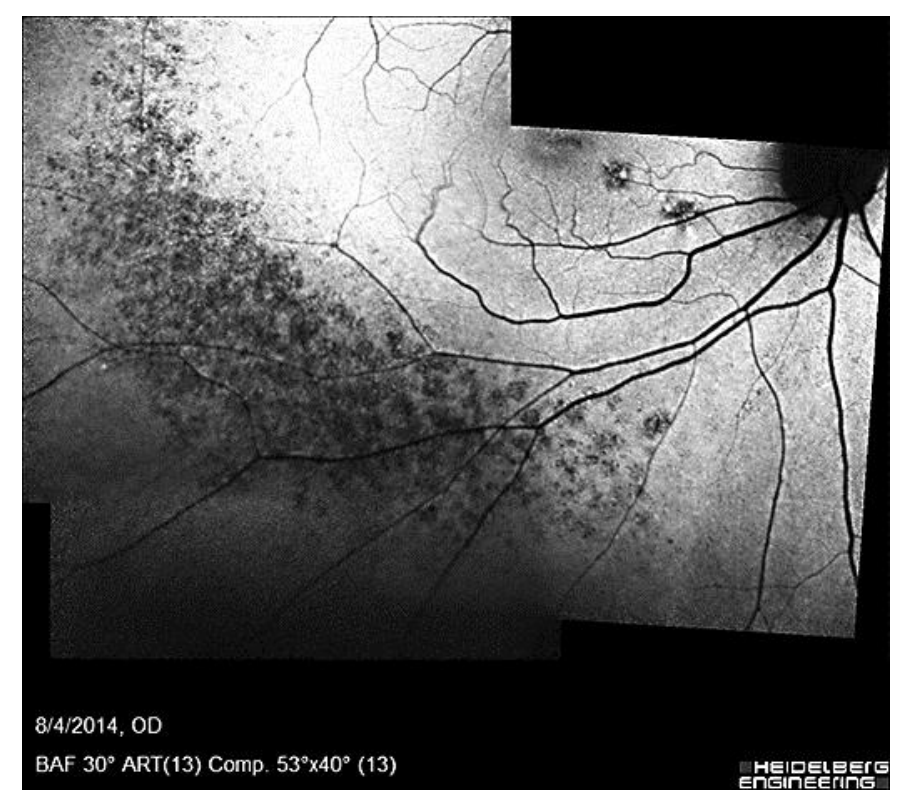

Fig. 2. FAF of the right eye showing foveal pigment disturbances and extensive retinal pigment epithelium changes outside the macula.

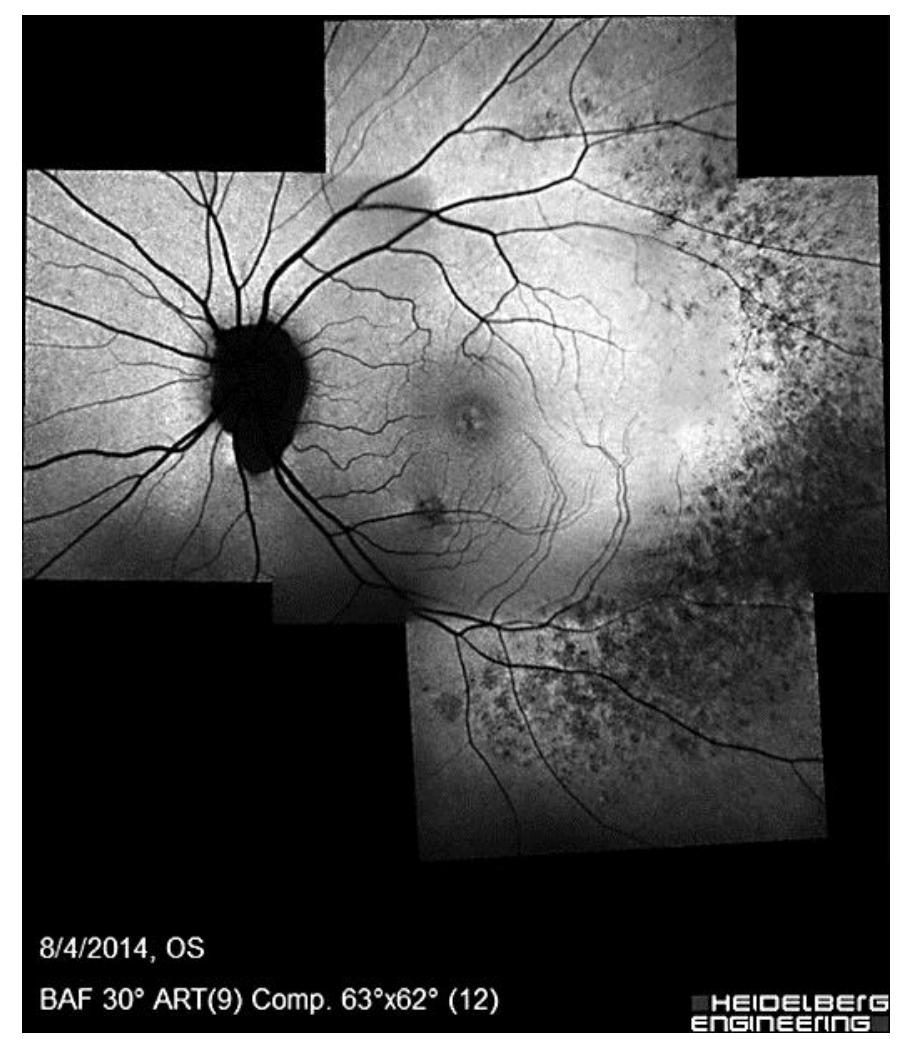

Fig. 3. FAF of the left eye showing foveal pigment disturbances and extensive retinal pigment epithelium changes outside the macula. 